\title{
Papa über 50? Kein Risikofaktor für Mutter und Kind
}

\begin{abstract}
Selbst wenn eine Schwangerschaft mithilfe der modernen Reproduktionsmedizin zustande kommt, werden die perinatalen Risiken für Mutter und Kind einer US-Studie zufolge offenbar nicht durch das Alter des Vaters beeinflusst.
\end{abstract}

W enig ist bislang darüber bekannt, inwieweit das Alter des Vaters den Ausgang einer Schwangerschaft beeinflusst. Einige Untersuchungen legen Auswirkungen des Alters auf die Spermienqualität und auf die Gesundheit des Kindes nahe. Um diese Unsicherheiten näher zu beleuchten, wurden in einer populationsbasierten, retrospektiven Kohortenstudie Daten zu allen Lebendgeburten in Ohio zwischen 2006 und 2012 analysiert und zudem der Einfluss der künstlichen Befruchtung (ART) auf das perinatale Outcome mit dem spontaner Konzeption verglichen. Insgesamt waren $3,7 \%$ der Neugeborenen mit Unterstützung der Reproduktionsmedizin entstanden. Bei 82,2\% der 1.034.552 Lebendgeburten war das Alter des Vaters bekannt. Die Spanne reichte von 12 bis 87 Jahre. 6.459 Männer gehörten der Altersgruppe 50-59 an, 602 Männer waren 60 oder älter. Je älter die Männer waren, desto häufiger bedienten sich die Paare der ART (bei den unter 30 -Jährigen $0,1 \%$, bei den über 60 -Jährigen $2,5 \%)$.
In der nicht adjustierten Analyse zeigten sich bei Männern in den höchsten Altersklassen die meisten Risiken. Das relative Risiko (RR) für genetische Krankheiten der Neugeborenen stieg von 40-49-jährigen Vätern auf das 2,84-Fache, von 50-59-Jährigen auf das 3,76-Fache und von Vätern ab 60 auf das 4,84-Fache gegenüber 30-39-Jährigen. Allerdings: Die meisten Männer über 40 hatten auch eine Partnerin jenseits der 35. Ging dieser Faktor in die Analyse ein, änderte sich das Bild: Nach Berücksichtigung des mütterlichen Alters (11-62 Jahre) und weiterer Risikofaktoren war kein signifikanter Einfluss des väterlichen Alters auf das perinatale Ergebnis mehr erkennbar. Weder zeigten sich in der adjustierten Analyse vermehrt Präeklampsien, Frühgeburten oder intensivmedizinische Behandlungen der Neugeborenen, noch fetale Wachstumsstörungen, kongenitale Anomalien oder genetische Erkrankungen. Zu dem gleichen Ergebnis kamen die Autoren auch dann, wenn sie den Einfluss des väterlichen Alters in Abhängigkeit

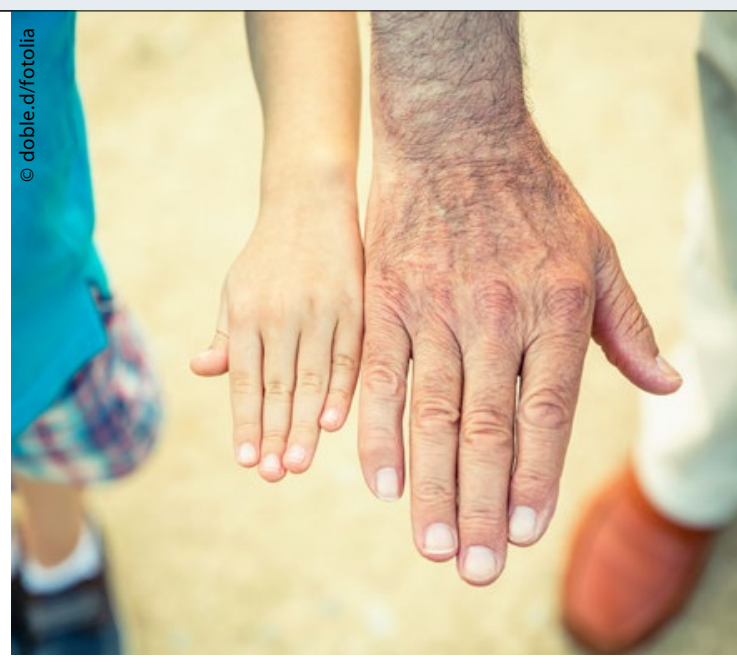

von der Zeugungsart betrachteten. Dabei wurden trotz der bekannten Risikoerhöhung durch die ART selbst keine nennenswerten Unterschiede beim perinatalen Outcome sichtbar, egal, ob die Kinder auf natürlichem Weg oder mithilfe der Reproduktionsmedizin entstanden waren. Laut dieser Daten stellt das Alter des Vaters kein unabhängiges Risiko für das perinatale Outcome dar. Vielmehr beeinflusse bei älteren Paaren, die sich noch zu einer Schwangerschaft entschließen, vor allem das Alter der Frau das Auftreten gesundheitlicher Komplikationen. Auch die Art der Zeugung scheine hier keine Rolle zu spielen. Dr. Christine Starostzik

Hurley E G et al. Influence of paternal age on perinatal outcomes. Am J Obstet Gynecol. 2017;217(5):566.e1-e6

\section{Mammakarzinom-Subtypen für Entscheidung zur Radiatio relevant}

\begin{abstract}
In einer Analyse der Phase-III-Studie EORTC 10994/BIG 1-00 wurden klinische und pathologische Faktoren für ein lokoregionäres Rezidiv (LRR) bei Brustkrebspatientinnen mit neoadjuvanter Chemotherapie untersucht, weil diese bei der Entscheidung über eine lokoregionale Bestrahlung helfen könnten.
\end{abstract}

\footnotetext{
In der Studie EORTC 10994/BIG 1-00 waren Frauen mit großen operablen oder lokal fortgeschrittenen Mammakarzinomen nach neoadjuvanter Chemotherapie (NAC) und Operation teilweise bestrahlt worden. Patientinnen mit Hormonrezeptor-positivem Tumor nahmen zudem adjuvant fünf Jahre Tamoxifen ein, bei HER2-positiven Tumoren erhielten sie adjuvant Trastuzumab. Nach median 4,4 Jahren hatten von 1.553 Patientinnen 67
}

ein LRR entwickelt (kumulative Inzidenz 4,9\%) - gegenüber früheren Ergebnissen wenige, eventuell wegen der Fortschritte bei der Radiotherapie, der Therapie mit Tamoxifen und Trastuzumab. Trotz niedriger Inzidenz ließen sich multivariat zwei wesentliche Prädiktoren für ein LRR identifizieren: Brustkrebs-Subtyp und pathologisches Ansprechen auf die NAC. Bei tripel-negativem Brustkrebs war das LRRRisiko gegenüber Luminal-A-Tumoren deutlich erhöht (HR 6,44; 95\%-KI 2,8314,69), ähnlich bei HER2-positivem Karzinom ohne Trastuzumab-Gabe (HR 6,26; 95\%-KI 2,81-13,93). Etwas günstiger, aber gegenüber Luminal-A-Tumoren noch deutlich erhöht, fiel das LRR-Risiko bei HER2-positiven Karzinomen mit Trastuzumab-Therapie aus (HR 3,37, 95\%-KI 1,10-10,34). Bei $\geq 4$ positiven Lymphknoten, war das LRR-Risiko mehr als verdoppelt. Die Resultate legen nahe, neben einem positiven nodalen Befund nach neoadjuvanter Chemotherapie auch den Mammakarzinom-Subtyp in die Entscheidung über eine adjuvante Strahlentherapie einzubeziehen. Friederike Klein

Gillon P et al. Factors predictive of locoregional recurrence following neoadjuvant chemotherapy in patients with large operable or locally advanced breast cancer: An analysis of the EORTC 10994/ BIG 1-00 study. Eur J Cancer. 2017;79:226-34 